\title{
Strategies for Optic Pathways Decompression for Extra-Axial Tumors or Intracranial Aneurysms: A Technical Note
}

\author{
Pier Paolo Mattogno ${ }^{1, *([)}$ Carmelo Lucio Sturiale ${ }^{1, *(1)} \quad$ Alessandro Rapisarda $^{1} \quad$ Alessandro Olivi $^{1}$ \\ Alessio Albanese ${ }^{1}$ \\ ${ }^{1}$ Department of Neurosurgery, Fondazione Policlinico Universitario \\ Agostino Gemelli IRCCS, Rome, Italy \\ J Neurol Surg A Cent Eur Neurosurg 2021;82:475-483.

\begin{abstract}
Address for correspondence Carmelo Lucio Sturiale, MD, PhD, Department of Neurosurgery, Fondazione Policlinico Universitario A. Gemelli IRCCS, Roma, 00168, Italy (e-mail: cropcircle.2000@virgilio.it).
\end{abstract}

\begin{abstract}
Background Different types of skull base tumors and intracranial aneurysms may lead to compression of the optic pathways. Since most of them are biologically benign conditions, the first aim of surgery is preservation of optic nerves rather than the oncologic radicality.

Materials and methods Based on the progressive technical refinements coming from our institutional experience of optic nerve compression from aneurysms and extra-axial tumors, we analyzed the surgical steps to release nerves and chiasm during tumor debulking and aneurysm clipping.

Results We distinguished vascular and tumor lesions according to the main direction of optic nerve compression: lateral to medial, medial to lateral, inferior to superior, and anterior to posterior. We also identified four fundamental sequential maneuvers to release the optic nerve, which are (1) falciform ligament (FL) section, (2) optic canal unroofing, (3) anterior clinoid process drilling, and (4) optic strut removal. The FL section is always recommended when a gentle manipulation of the optic nerve is required. Optic canal unroofing is suggested in case of lateral-to-medial compression (i.e., clinoid meningiomas), medial-to-lateral compression (i.e., tuberculum sellae meningiomas), and inferior-to-superior compression (i.e., suprasellar lesions). Anterior

Keywords

- optic nerve

- optic pathways

- decompression

- falciform ligament

- optic canal clinoidectomy and optic strut removal may be necessary in case of lateral-to-medial compression from paraclinoid aneurysms or meningiomas.

Conclusions Preservation of the visual function is the main goal of surgery for tumors and aneurysms causing optic nerve compression. This mandatory principle guides the approach, the timing, and the technical strategy to release the optic nerve, and is principally based on the direction of the compression vector.
\end{abstract}

\section{Introduction}

Optic pathways are extremely fragile nervous structures coursing in the cisternal space of the anterior skull base close to other important neurovascular elements. Several extra-

Alphabetically ordered first coauthors

received

April 25, 2020

accepted after revision

August 25, 2020

published online

February 28, 2021 axial tumors and vascular malformations may determine a compression of the optic pathways at the level of nerve, chiasm, or posterior tract.

However, most pathologies involving this anatomical area are benign, and usually determine a visual impairment due to optic nerve compression rather than invasion.

(c) 2021. Thieme. All rights reserved. Georg Thieme Verlag KG, Rüdigerstraße 14, 70469 Stuttgart, Germany
DOI https://doi.org/ 10.1055/s-0040-1720991. ISSN 2193-6315. 
Therefore, in these cases, the main aim of surgery is the preservation of visual function rather than the gross total resection of the lesion, especially in the light of modern radiosurgery and endovascular techniques.

Several surgical strategies have been proposed to release the optic nerves from extrinsic compressions, but a proper algorithm has not yet been described. This should be designed according to the biology of the disease, the area of origin, and the direction of growth and compression.

In this article, we discuss the most frequent pathologies involving the optic nerves associated with visual impairment, and design an algorithmic strategy for optic pathway decompression.

\section{Material and Methods}

Based on the progressive technical refinements from our institutional experience of optic nerve compression from aneurysms and extra-axial tumors, we analyzed the surgical steps to release nerves and chiasm during tumor debulking and aneurysm clipping.

Then, we identified some fundamental key points and designed an algorithm concerning approach, microsurgical dissection, falciform ligament (FL) opening, optic nerve mobilization, tumor removal, and aneurysm clipping technique discussing the safety precautions that we adopted in different surgeries.

Review by the institutional review board was not necessary, because we included only anonymous data archived in our institutional database with the informed consent that the patients signed at the time of surgery.

\section{Results}

We identified two major families of extrinsic lesions compressing the optic pathways: vascular malformations and extra-axial tumors. Moreover, we divided these pathologies according to the epicenter of the origin and direction of optic pathway compression:
- Lesions determining a lateral-to-medial compression, such as clinoid meningiomas and paraclinoid aneurysms.

- Lesions determining a medial-to-lateral compression, such as tuberculum sellae meningiomas.

- Lesions determining an inferior-to-superior compression (often in combination with a medial-to-lateral compression), such as sellar tumors (adenomas and cystic craniopharyngiomas, epidermoids, etc.).

- Lesions determining an anterior-to-posterior compression, such as olfactory groove meningiomas.

We also recognized four fundamental maneuvers useful to unlock and, in case of extreme necessity, mobilize the optic nerve:

- FL section.

- Optic canal unroofing.

- Anterior clinoid process drilling.

- Optic strut removal.

These different maneuvers are summarized in - Table 1 according to their necessity in the different pathologies examined.

\section{Falciform Ligament Section}

The FL is a dural duplication above the planum sphenoidale that forms the soft component of the roof of the optic foramen. The FL locks the optic nerve where it enters the optic canal, and may act as a knifelike edge in case of expansive lesions that determine an optic nerve stretching in a caudocranial sense. ${ }^{1}$ The FL can be easily identified, lifted up through a micro-hook above the optic nerve plane and then cut with microscissors, paying attention to avoid optic



\section{Optic Canal Unroofing}

The optic foramen represents the door of the optic canal in the sphenoid bone, medially bounded by the body and laterally by the lesser wing of the sphenoid.

Table 1 Summary of different directions of compression caused by vascular and neoplastic pathologies and the surgical steps to "unlock" the optic nerve

\begin{tabular}{|c|c|c|c|c|c|c|}
\hline $\begin{array}{l}\text { Direction of } \\
\text { compression }\end{array}$ & Pathology & $\begin{array}{l}\text { Bilateral optic } \\
\text { pathway } \\
\text { involvement }\end{array}$ & $\begin{array}{l}\text { Falciform } \\
\text { ligament } \\
\text { section }\end{array}$ & $\begin{array}{l}\text { Optic } \\
\text { canal } \\
\text { unroofing }\end{array}$ & $\begin{array}{l}\text { Anterior clinoid } \\
\text { process drilling }\end{array}$ & $\begin{array}{l}\text { Optic } \\
\text { strut } \\
\text { removal }\end{array}$ \\
\hline \multirow[t]{2}{*}{ Lateral to medial } & clinoid meningiomas & no & 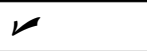 & レ & 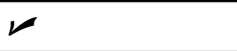 & - \\
\hline & paraclinoid aneurysms & no & $\nu$ & レ & $\nu$ &  \\
\hline \multirow[t]{2}{*}{ medial to lateral } & $\begin{array}{l}\text { tuberculum sellae } \\
\text { meningiomas }\end{array}$ & yes & $レ$ & $\boldsymbol{V}$ & - & - \\
\hline & $\begin{array}{l}\text { Diaphragma sellae } \\
\text { meningiomas }\end{array}$ & yes & 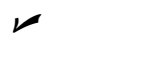 & レ & - & - \\
\hline \multirow{2}{*}{$\begin{array}{l}\text { inferior to superior } \\
\text { (and medial to lateral) }\end{array}$} & Craniopharyngiomas & yes & $\nu$ & - & - & - \\
\hline & Pituitary macroadenomas & yes & レ & - & - & - \\
\hline anterior to posterior & $\begin{array}{l}\text { Olfactory groove } \\
\text { meningiomas }\end{array}$ & yes & 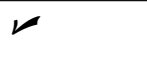 & $\boldsymbol{V}$ & - & - \\
\hline
\end{tabular}




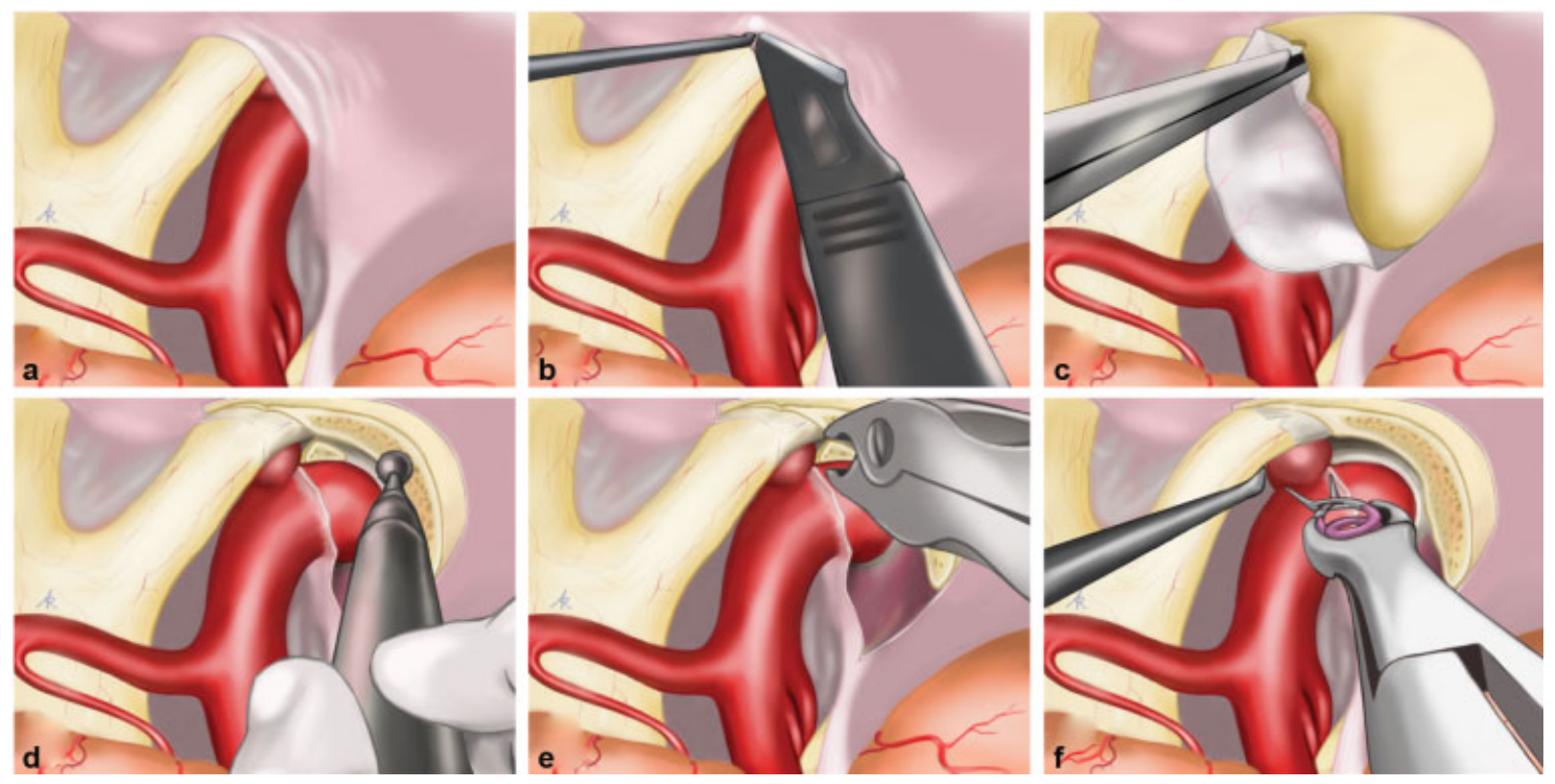

Fig. 1 Procedure of optic nerve mobilization for paraclinoid aneurysm clipping. (a) The aneurysm appears covered by the right optic nerve. (b) After a sharp cutting of the falciform ligament, (c) optic canal unroofing is performed with a Kerrison rongeur and (d) the anterior clinoid process is drilled. (e) After the partial removal of the optic strut, (f) the optic nerve is gently mobilized to allow the aneurysm exclusion.

The combination of optic canal unroofing and anterior clinoidectomy was introduced by Dolenc as a preliminary surgical step to manage paraclinoid aneurysms and skull base tumors. ${ }^{2}$ It can provide wider surgical corridors and early optic nerve decompression and mobilization. ${ }^{1,3}$ The unroofing can be performed with a Kerrison rongeur after the reflection of the dural flap extending from the FL section over the optic nerve as a protection (-Fig. 1c).

High-speed drills may be used in case of hyperostotic lesions, paying attention to avoid optic nerve heat injury. ${ }^{4,5}$

Ultrasonic bone aspirator may be alternatively used, which reduces these potential complications. ${ }^{6}$

\section{Anterior Clinoid Process Drilling and Optic Strut Removal}

The anterior clinoid process is a medial projection of the lesser sphenoid wing, bridged to the sphenoid by the optic strut, and forming the lateral wall of the optic canal. Its inferomedial surface is in contact with the ophthalmic segment of the internal carotid artery (ICA), which is enveloped by two dural rings. Removal of the anterior clinoid process, and its extension to the strut, is a crucial step in the management of ophthalmic aneurysms and parasellar/cavernous sinus lesions.

Anterior clinoidectomy can be performed with intradural or extradural techniques, and allows a wider exposure of the ICA and the superior surface of the cavernous sinus. After a pterional craniotomy, the greater sphenoid wing is drilled until the orbitotemporal periosteal fold (OTPF) is reached. After transection of the OTPF, the superior aspect of the clinoid and lateral wall of the cavernous sinus can be exposed peeling away the dura propria. Clinoid drilling starts from its lateral aspect until exposing the optic strut inferomedial to the optic nerve sheath. The strut can be microfractured and removed en block (-Fig. 1e-f).
Concerning the intradural technique, after frontotemporal dural opening and basal cistern detention, a dural incision is performed over the clinoid process and the flap posteriorly reversed using a microdissector. Then, the clinoid is drilled until the strut is exposed.

When necessary, the optic strut can be removed and the distal dural ring opened to obtain a circumferential optic nerve decompression.

\section{Lesions Determining a Lateral-to-medial Compression}

In case of large clinoid meningiomas, there is usually a lateral-to-medial compression of the ipsilateral optic nerve without direct invasion (-Fig. 2a). Generally, the arachnoid layer between the tumor capsule and the optic nerve remains identifiable. Through the pterional corridor, tumor mass and hyperostotic clinoid are interposed between the surgical window and the optic nerve. Thus, after a partial tumor debulking, it is necessary to drill the clinoid process, cut the FL, and sometimes unroof the optic canal before any optic nerve manipulation.

Similarly, in case of paraclinoid aneurysms, the direction of optic nerve compression is lateral to medial, but there is not sphenoidal hyperostosis. Therefore, the FL section may be sufficient to gently mobilize the optic nerve and expose the aneurysm neck for clipping. In case of more proximal aneurysms, such as those originating from the ophthalmic ICA, anterior clinoid drilling is necessary to obtain proximal control.

\section{Lesions Determining a Medial-to-Lateral Compression} Tuberculum sellae meningiomas generally involve optic nerves bilaterally, due to optic canal invasion and reactive hyperostosis. In case of larger tumors, the chiasm can also be 

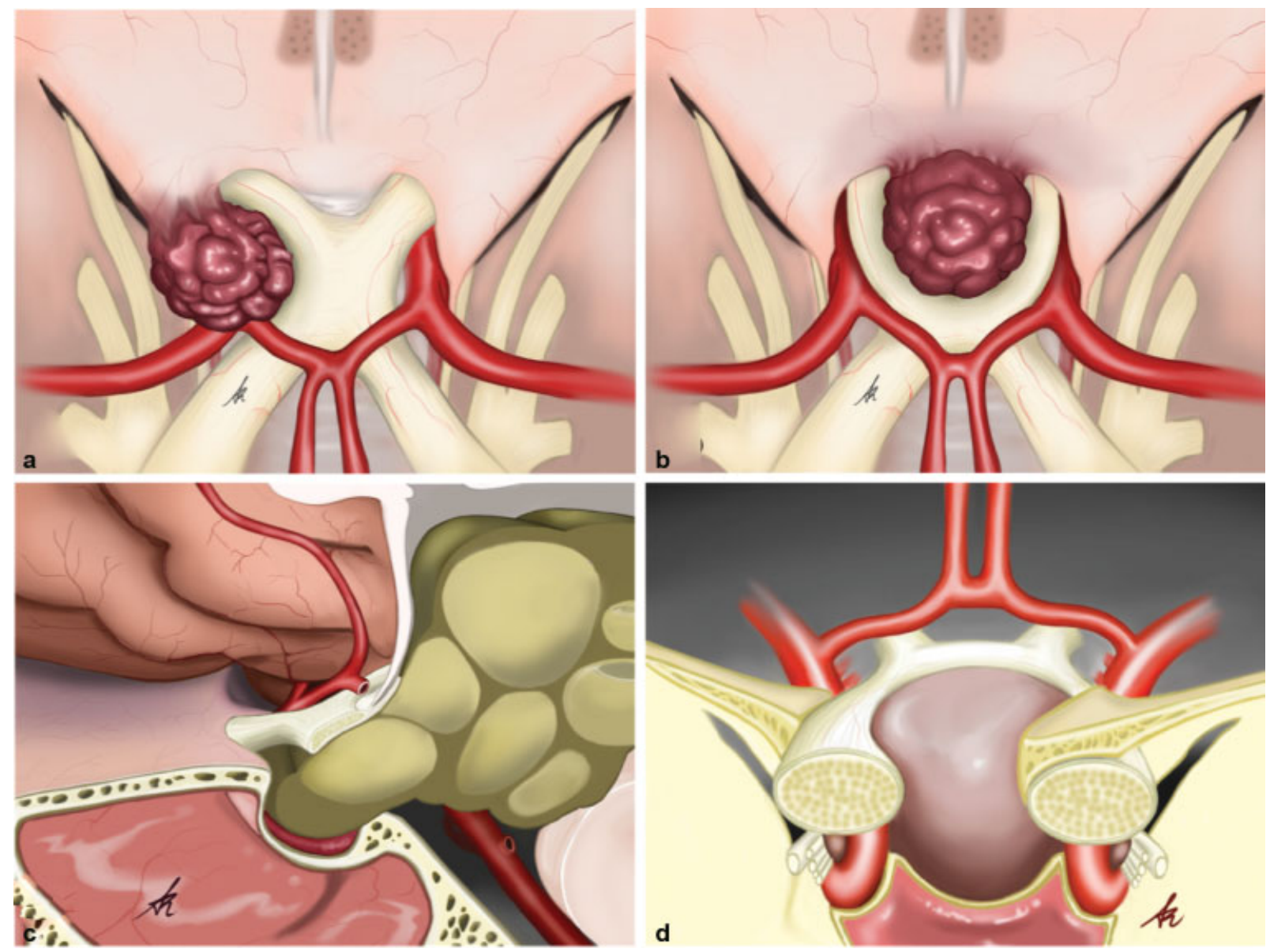

Fig. 2 Demonstration of the presence of (a) an extra-axial tumor originating from the left anterior clinoid process determining a lateral-tomedial dislocation of the ipsilateral optic nerve (ON), (b) an extra-axial lesion originating from tuberculum/diaphragma sellae determining a bilateral medial-to-lateral ON dislocation, (c) a lesion originating from the pituitary stalk and extending toward the third ventricle and the interpeduncular cistern with a prefixed chiasm and an inferior-to-superior ON dislocation, and (d) a lesion originating from the pituitary gland and extending toward the chiasmatic cistern, bilateral dislocation of the ONs in an inferior-to-superior and medial-to-lateral direction.

involved ( - Fig. $2 \mathbf{b}$ ). The growth vector of the lesion determines a bilateral medial-to-lateral optic nerve dislocation, but the arachnoid plane between the tumor and the optic nerve usually remains preserved. Sometimes, a prevalent unilateral visual field cut may be observed in case of asymmetric invasion of optic canals. The growth vector of the lesion may sometimes also determine an inferior-to-superior dislocation of the chiasm, which typically determines a bitemporal hemianopia. Regardless of whether a trans-sylvian or subfrontal approach is preferred, releasing the optic nerves through the FL section and unroofing of the optic canal is mandatory before any manipulation during tumor debulking. In particular, in case of larger tumors, optic nerve identification and dissection represent the most risky maneuvers, as they can be laterally displaced and thinned by chronic compression. Generally, optic nerves are identified more easily where they enter the optic canal, which also represents the main point of optic nerve anchoring and compression due to the presence of a rigid FL. Finally, during optic nerve dissection, it is important to pay attention to the course of the ICA, which could be similarly displaced and unrecognizable.

\section{Lesions Determining the Combination of a Medial-to- Lateral and Inferior-to-Superior Compression}

Large sellar or suprasellar lesions such as craniopharyngiomas or macroadenomas may cause a medial-to-lateral dislocation of the optic nerves (-Fig. $\mathbf{2 c}$, d). At the same time, these tumors can deform the optic pathways on the sagittal plane by stretching the optic nerves (prefixed chiasms), the optic tracts (postfixed chiasms), or both (central compression). These tumors can be reached through the subfrontal or sylvian corridors, but a preliminary FL section is often necessary to obtain sufficient optic nerve freedom, allowing safe mobilization and sufficient surgical window to debulk the lesion.

Pituitary adenomas going beyond the sellar diaphragm can cause optic nerve and chiasm compression, with vectors directed upward and laterally. In the vast majority of cases, these lesions can be managed through an endonasal approach, but in case of lateral extension, craniotomy may be the preferred approach. Adenomas are generally soft and/or friable and usually respect the arachnoid plane with other neurovascular structures. Their gross total resection is achievable, but a gentle mobilization of the ipsilateral optic nerve is usually necessary to widen the surgical corridor. Generally, 
the FL section alone is sufficient to gain the necessary degree of optic nerve freedom to avoid its damage.

\section{Lesions Determining an Anterior-to-Posterior Compression}

Olfactory groove meningiomas are anterior extra-axial skull base tumors that can displace and compress optic pathways. Generally, they are of benign nature and respect the arachnoid plane, but can cause visual impairments due to optic canal roof hyperostosis. They can be approached through pterional or frontal craniotomy according to the surgeon preference and tumor extension. In the first case, the surgical window allows exposure of the ipsilateral optic nerve that is laterally displaced. Thus, the optic nerve needs to be mobilized to allow tumor debulking, and the FL section and unroofing of the optic canal in case of hyperostosis represent preliminary mandatory maneuvers.

Moreover, at the end of the debulking, it is crucial to recognize the arachnoid plane that separates the tumor capsule from the contralateral optic nerve to avoid its accidental injury.

In the subfrontal approach, the gross total debulking precedes optic nerve identification and dissection, which occurs only at the end of tumor removal. It is necessary to assess on a case-by-case basis to decide whether one or both optic nerves should be mobilized to remove the last portion of the lesion to perform the preliminary maneuvers of optic nerve unlocking.

\section{Discussion}

Different surgical approaches have been proposed for optic nerve release in case of extrinsic compression. The transcranial routes represent the first choice, allowing a circumferential decompression. ${ }^{7,8}$ On the other hand, minimally invasive techniques, such as endonasal and transorbital approaches, have been increasingly used in the last decades. ${ }^{9-14}$

The goals of surgical management of the parasellar tumors should be optic nerve decompression to limit visual loss and gross total resection of the tumor for a better oncologic outcome. In general, pursuing a larger extension of the resection may require a major optic nerve manipulation. In all these cases, preservation of the optic pathways is crucial and surgical strategy should primarily take into account the concepts of "unlock the structures (optic nerves)" and "surgical freedom (of the optic nerves)," meaning optic nerve manipulation should be performed only after having them adequately released from the surrounding osteodural structures anchoring them to the anterior skull base, to avoid excessive stretching and damage (-Fig. 3 ).
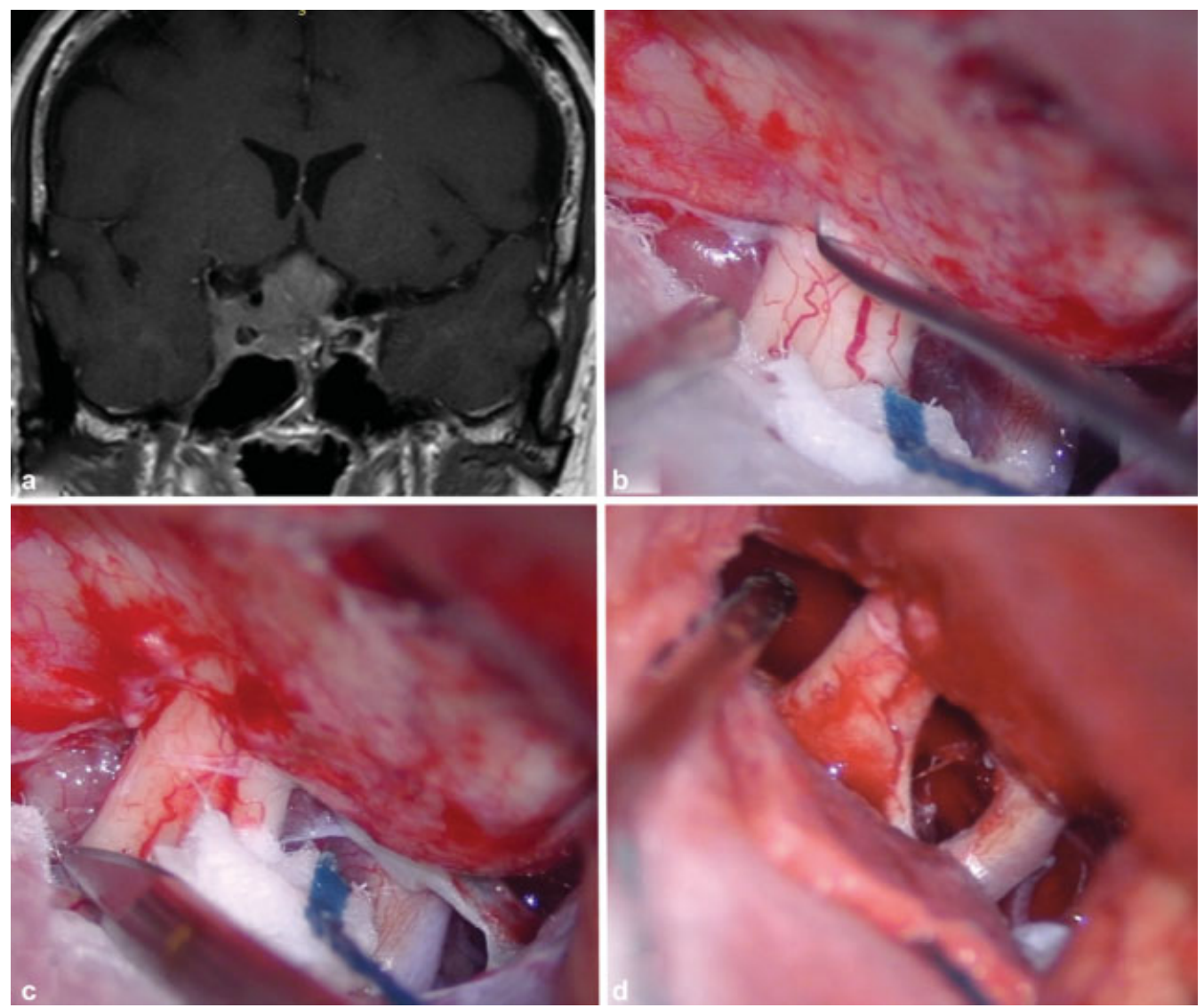

Fig. 3 Case of a 68-year-old patient affected by a nonsecreting pituitary adenoma with right intracavernous component (a). Due to the progressive tumor growth associated with visual impairment in the right eye, the patient underwent tumor resection through a right subfrontal approach. (b) The falciform ligament is immediately identified and (c) cut before any tumor manipulation. (d) At the end of the procedure, the tumor lying under the optic nerve plain is completely removed with minimal nerve traction. Cutting the falciform ligament guarantees the necessary mobility of the nerve during the tumor debulking unlocking a major anchor point. 


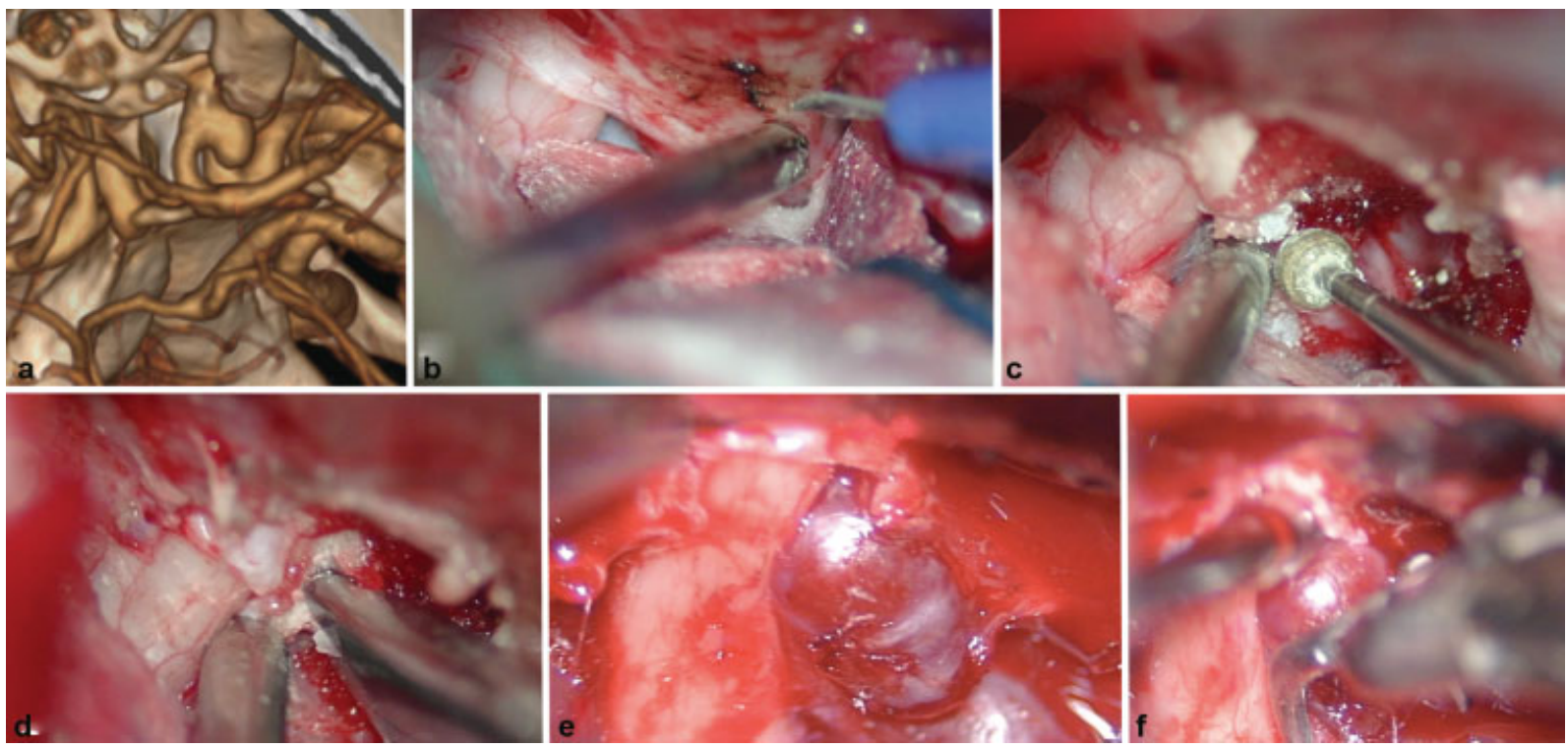

Fig. 4 Case of a 45-year-old patient with a clinical history of progressive visual disturbance in the right eye associated with chronic headache. (a) Radiologic examinations documented an ophthalmic tract internal carotid artery (ICA) aneurysm compressing the optic nerve. The patient underwent surgical clipping of the aneurysm through a right lateral supraorbital approach. (b) After the basal cistern dissection, the dura covering the anterior clinoid process is cut along with the falciform ligament; then, (c) the process is drilled until the exposure of the optic strut, (d) which is removed with a Kerrison rongeur. (e) Finally, the aneurysm neck is exposed, (f) allowing the exclusion of the malformation with a minimal traction on the optic nerve. See video.

Surgery for paraclinoid aneurysms needs to deal with numerous critical neurovascular structures of the basal cistern, such as ICA, optic nerves, oculomotor nerves, anterior cerebral artery, and posterior communicating artery. Clipping of these aneurysms often requires ipsilateral optic nerve manipulation for complete exposure of the aneurysm neck and proximal control. This maneuver implies a certain risk of optic nerve damage and postoperative visual impairment (-Fig. 4).

Silva et al reported a high rate of visual improvement (58\%) after paraclinoidal aneurysm clipping. ${ }^{15}$

Ophthalmic ICA aneurysms and superior hypophyseal artery aneurysms, especially $>1 \mathrm{~cm}$, may cause optic pathway compression. The dislocation is usually superomedially directed against the roof of the optic canal and the FL ( - Fig. 1d, e). Even though proximal control can be secured at the extracranial ICA, a certain optic nerve manipulation is necessary to expose the aneurysm neck. ${ }^{16-18}$ Thus, FL opening, unroofing of the optic canal, and drilling of the anterior clinoid process and optic strut can be necessary before the aneurysm clipping. The larger the aneurysm size, the greater the usefulness of these maneuvers. In particular, optic strut removal and opening of the distal dural ring may be required in selective cases to improve lateral mobilization of the ICA.

Posterior communicating artery aneurysms originate from the ICA segment between the origin of the posterior communicating artery and the ICA bifurcation and point toward the lateral carotid cistern. ${ }^{16}$ Generally, they do not result in direct compression of the optic pathways unless they are giant size. Nevertheless, in some circumstances, optic nerve manipulation may be necessary to guarantee proximal control for any temporary clipping, especially if the proximal ICA is $<3 \mathrm{~mm}{ }^{16,17}$
Carotid cave aneurysms originate from the medial wall of the paraclinoid ICA, between the distal dural ring and the origin of the ophthalmic artery, and represent 7 to $17 \%$ of all paraclinoid aneurysms. ${ }^{19}$ Because of their medial projection, clippings should be done in such a way that the optic nerve is mobilized medially and the ICA laterally. In these cases, all the described maneuvers to optic nerve unlocking need to be performed preliminarily to reduce the risk of visual disturbances after surgery. ${ }^{19-21}$

Anterior clinoid meningiomas represent one of the tumors that most frequently determine optic nerve/chiasm compression during their natural history. Their growth vector is directed upward to the sylvian fissure, differently from the greater sphenoidal wing meningiomas, which generally grow toward the medial aspect of the temporal lobe ( $\mathbf{- F i g . ~ 5 ;}$ -Video 1). Even the latter, however, may sometimes present medial extension with ipsilateral optic nerve or chiasm compression. An anterior clinoid hyperostosis is often associated with these skull base tumors, and may contribute to optic pathway compression causing visual impairment with a mechanism of chronic ischemia and demyelination. ${ }^{22}$

\section{Video 1:}

Two exemplificative cases of optic pathways decompression from (a) a optic foramen meningioma and (b) an ophthalmic carotid artery aneurysm. (a) Through a subfrontal way, basal cisterns are progressively opened obtaining CSF drainage and brain relaxation. Then, the meningioma appears immediately visible under the plane of the optic nerve that in turn appears pushed upward. The tumor is also 

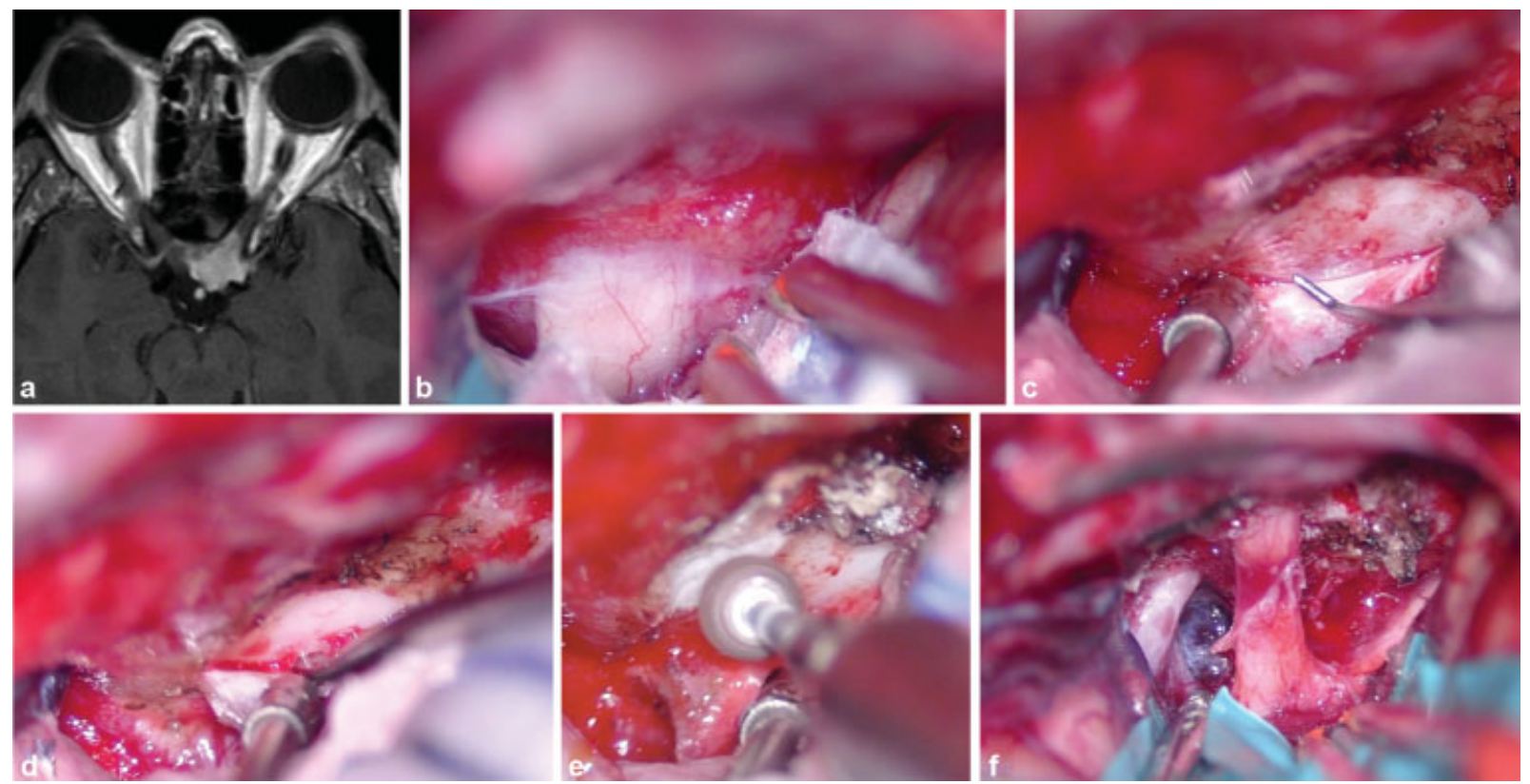

Fig. 5 Case of a 57-year-old patient with a clinical history of severe visual impairment in the left eye. (a) A brain magnetic resonance imaging with gadolinium documented meningioma invading the left optic canal. The patient underwent tumor removal through the left pterional craniotomy. (b) After basal cistern dissection, the tumor appears to be emerging from the optic canal enveloping the optic nerve. (c,d) The falciform ligament is cut along with the dura mater covering the anterior clinoid process and (e) the clinoid bone is drilled until the exposure of the optic strut. (f) The tumor is then completely resected decompressing the cisternal and the intracanal tract of the optic nerve. See video.

bilaterally extended both towards the carotid-optic and the interoptic cisterns. The falciform ligament appears thickened and hyperemic in relation to the meningioma vascular supply. After the falciform ligament section, the roof of the optic canal is drilled, thus enhancing the degree of mobility of the optic nerve during the tumor removal that implies an unavoidable, although minimal, nerve manipulation. At the end of procedure the optic nerve appears decompressed, but the hyperemic signs of a previous sufferance are still evident. (b) After the basal cisterns dissection, the CSF release and the brain detention, the optic nerve and the internal carotid artery at the supraclinoid tract are immediately evident. In order to expose the aneurysm neck and obtaining the control on the proximal carotid artery, it is necessary cutting and flipping the dura mater covering the anterior clinoid process, which is in turn drilled until the distal dural ring is seen. Then, the aneurysm can be excluded with a single titanium clip.Online content including video sequences viewable at: https://www. thieme-connect.com/products/ejournals/html/ 10.1055/s-0040-1720991.

From a surgical point of view, we recognize three different neoplastic components: the cisternal portion, which protrudes superiorly in the parasellar cisterns; the cavernous/ carotid portion that extends inferiorly; and the portion projecting inferomedially, which usually intersects the optic canal determining optic nerve compression and dislocation. ${ }^{23}$
As these tumors are usually benign, the first goal of surgery is optic nerve decompression to preserve residual visual function. A standard pterional approach is usually preferred by most of the authors, and an extradural clinoidectomy may help the initial deafferentation. The intradural phase starts with the maneuvers necessary to unlock the optic nerve, such as cutting the FL and optic canal unroofing, which are preliminary to any optic nerve manipulation. ${ }^{23}$

Tuberculum sellae meningiomas usually present an upward extension causing compression and dislocation of the chiasm. They grow in the interoptic cistern and may push the optic nerves and the ICAs laterally, and posteriorly dislocate the hypothalamus, infundibulum, and the pituitary stalk, sometimes with severe clinical implications. They involve the optic canal by a lateral extension besides growing with a posterosuperior vector, then causing a double compression on the optic pathways. ${ }^{24}$

The transcranial microsurgery was the standard treatment until the emergence of the extended endoscopic endonasal approach. ${ }^{24}$ Although there is no clear evidence to support the superiority of either approach, the risk of a worsening vision seems higher in craniotomy in certain series. ${ }^{25}$ However, in these series routine use of the preliminary maneuvers to unlock the optic nerve is not always reported.

Craniopharyngiomas generally originate in the hypothalamic-pituitary region and present different patterns of suprasellar extension such as preinfundibular (type I); transinfundibular (type II); retroinfundibular (type III) with their variants (IIIa: extended into the third ventricle and IIIb: extended into the interpeduncular cistern); and isolated intraventricular (type IV). ${ }^{26}$ They are commonly associated 
with visual symptoms, especially in the cystic forms. The growth vector of craniopharyngiomas varies in relation to the type of extension, but generally causes upward stretching of the optic pathways against the FL and the roof of the optic canal. The optic chiasm can be displaced as prefixed, postfixed, or present a central compression. ${ }^{27}$

The extended endoscopic endonasal approach has become the most widely used method for tuberculum sellae meningiomas. This approach allows us to operate along the major axis of the lesion and to perform tumor debulking with decompression of optic pathways without "working across the nerves." 28 In case of huge size or third ventricle extension, the transcranial approach may be necessary to debulk the lesion and to decompress the optic pathway. In these cases, the preliminary maneuvers to unlock the optic nerve, especially the incision of the FL and sometimes opening the optic canal, are mandatory before starting the tumor debulking, which may require the optic nerve manipulation.

Pituitary adenomas represent the most common pathology of the diencephalon-pituitary region and often cause visual disturbances related to the compression of the optic chiasm. The gold standard for surgical management of pituitary adenomas is currently represented by the transsphenoidal endonasal endoscopic technique, which in the past 20 years, thanks to the numerous technical innovations and the growing experience, has progressively replaced the craniotomy and microsurgical transsphenoidal approach in most cases with excellent results in terms of clinical and radiologic outcome. However, in some selected cases ( $1-4 \%)$, as in adenomas with lateral extension to the plane of the carotid arteries or in case of particularly complex giant adenomas with carotid/cavernous sinus encasing, the microsurgical craniotomy is still the preferred approach. ${ }^{29,30}$

In other anterior skull base lesions, the transcranial approach as effective as the endoscopic approach. ${ }^{25,31}$

The pterional frontal temporal approach with subfrontal extension allows immediate control of the ICA and the optic nerve, allowing a release of the nerve through the opening of the FL; in this way, safe surgical removal can be performed, exploring the interoptic cistern and the suprasellar space having direct control of the parasellar neurovascular structures.

\section{Conclusion}

In the case of oncologic or vascular pathology that causes a compression of the optic pathways, optic nerve decompression to avoid a visual impairment represents the first and most important outcome of surgery. Surgical radicality often represents a secondary objective mainly because the majority of these lesions are biologically benign.

Therefore, all the surgical maneuvers that unlock the optic nerves and allow gentle mobilization of these nerves during surgery should always precede tumor debulking or aneurysm neck dissection and clipping.

Conflict of Interest

None declared.

\section{References}

1 Woodall MN, Alleyne CH Jr. Carotid-falciform optic neuropathy: microsurgical treatment. World Neurosurg 2017;104:372-375

2 Dolenc VV.A combined epi- and subdural direct approach to carotidophthalmic artery aneurysms. J Neurosurg 1985;62(05):667-672

3 Fukuda H, Evins AI, Burrell JC, Iwasaki K, Stieg PE, Bernardo A. The meningo-orbital band: microsurgical anatomy and surgical detachment of the membranous structures through a frontotemporal craniotomy with removal of the anterior clinoid process. J Neurol Surg B Skull Base 2014;75(02):125-132

4 Korosue K, Heros RC. "Subclinoid" carotid aneurysm with erosion of the anterior clinoid process and fatal intraoperative rupture. Neurosurgery 1992;31(02):356-359, discussion 359-360

5 Chang DJ. The "no-drill" technique of anterior clinoidectomy: a cranial base approach to the paraclinoid and parasellar region. Neurosurgery 2009;64(03):ons96-ons105, discussion ons105-ons106

6 Golub JS, Weber JD, Leach JL, et al. Feasibility of the ultrasonic bone aspirator in retrosigmoid vestibular schwannoma removal. Otolaryngol Head Neck Surg 2015;153(03):427-432

7 Caporlingua A, Prior A, Cavagnaro MJ, et al. The intracranial and intracanalicular optic nerve as seen through different surgical windows: endoscopic versus transcranial. World Neurosurg 2019;124:522-538

8 Bernardo A, Evins AI, Mattogno PP, Quiroga M, Zacharia BE. The orbit as seen through different surgical windows: extensive anatomosurgical study. World Neurosurg 2017; 106:1030-1046

9 Beer-Furlan A, Abi-Hachem R, Jamshidi AO, Carrau RL, Prevedello DM. Endoscopic trans-sphenoidal surgery for petroclival and clival meningiomas. J Neurosurg Sci 2016;60(04):495-502

10 Kong DS, Hong CK, Hong SD, et al. Selection of endoscopic or transcranial surgery for tuberculum sellae meningiomas according to specific anatomical features: a retrospective multicenter analysis (KOSEN-002). J Neurosurg 2018;130(03):838-847

11 Zada G, Fredrickson VL, Wrobel BB. Extended endoscopic endonasal approach for resection of tuberculum sellae meningioma. Neurosurg Focus 2017;43(VideoSuppl2):V2

12 Ditzel Filho LF, Prevedello DM, Jamshidi AO, et al. Endoscopic endonasal approach for removal of tuberculum sellae meningiomas. Neurosurg Clin N Am 2015;26(03):349-361

13 Lobo B, Zhang X, Barkhoudarian G, Griffiths CF, Kelly DF. Endonasal endoscopic management of parasellar and cavernous sinus meningiomas. Neurosurg Clin N Am 2015;26(03):389-401

14 Almeida JP, Omay SB, Shetty SR, et al. Transorbital endoscopic eyelid approach for resection of sphenoorbital meningiomas with predominant hyperostosis: report of 2 cases. J Neurosurg 2018; 128(06):1885-1895

15 Silva MA, See AP, Dasenbrock HH, Patel NJ, Aziz-Sultan MA. Vision outcomes in patients with paraclinoid aneurysms treated with clipping, coiling, or flow diversion: a systematic review and metaanalysis. Neurosurg Focus 2017;42(06):E15

16 Sturiale CL, Marchese E, Puca A, Olivi A, Albanese A. Surgical treatment of posterior communicating artery aneurysms: hints and precautions for young cerebrovascular surgeons. J Neurol Surg A Cent Eur Neurosurg 2019;80(03):205-212

17 Kamide T, Tabani H, Safaee MM, Burkhardt JK, Lawton MT. Microsurgical clipping of ophthalmic artery aneurysms: surgical results and visual outcomes with 208 aneurysms. J Neurosurg 2018;129(06):1511-1521

18 Nanda A, JavalkarV. Microneurosurgical management of ophthalmic segment of the internal carotid artery aneurysms: single-surgeon operative experience from Louisiana State University, Shreveport. Neurosurgery 2011;68(02):355-370, discussion 370-371

19 Kobayashi S, Kyoshima K, Gibo H, Hegde SA, Takemae T, Sugita K. Carotid cave aneurysms of the internal carotid artery. J Neurosurg 1989;70(02):216-221 
20 Tabani H, Yousef S, Burkhardt JK, Gandhi S, Benet A, Lawton MT. Microsurgical clipping of an unruptured carotid cave aneurysm: 3-dimensional operative video. World Neurosurg 2017;104:1045. e3

21 Okudera H, Kobayashi S, Kyoshima K, Nitta J. Ring clip with laterally curved blades for carotid cave aneurysm. Neurosurgery 1996;39(03):614-616

22 Pamir MN, Belirgen M, Ozduman K, Kiliç T, Ozek M. Anterior clinoidal meningiomas: analysis of 43 consecutive surgically treated cases. Acta Neurochir (Wien) 2008;150(07):625-635, discussion 635-636

23 Sughrue M, Kane A, Rutkowski MJ, Berger MS, McDermott MW. Meningiomas of the anterior clinoid process: is it wise to drill out the optic canal? Cureus 2015;7(09):e321

24 Magill ST, Morshed RA, Lucas CG, et al. Tuberculum sellae meningiomas: grading scale to assess surgical outcomes using the transcranial versus transsphenoidal approach. Neurosurg Focus 2018;44(04):E9

25 Lu VM, Goyal A, Rovin RA. Olfactory groove and tuberculum sellae meningioma resection by endoscopic endonasal approach versus transcranial approach: a systematic review and meta-analysis of comparative studies. Clin Neurol Neurosurg 2018;174:13-20
26 Kassam AB, Gardner PA, Snyderman CH, Carrau RL, Mintz AH, Prevedello DM. Expanded endonasal approach, a fully endoscopic transnasal approach for the resection of midline suprasellar craniopharyngiomas: a new classification based on the infundibulum. J Neurosurg 2008;108(04):715-728

27 Prieto R, Pascual JM, Barrios L. Optic chiasm distortions caused by craniopharyngiomas: clinical and magnetic resonance imaging correlation and influence on visual outcome. World Neurosurg 2015;83(04):500-529

28 Wannemuehler TJ, Rubel KE, Hendricks BK, et al. Outcomes in transcranial microsurgery versus extended endoscopic endonasal approach for primary resection of adult craniopharyngiomas. Neurosurg Focus 2016;41(06):E6

29 Couldwell WT. Transsphenoidal and transcranial surgery for pituitary adenomas. J Neurooncol 2004;69(1-3):237-256

30 Youssef AS, Agazzi S, van Loveren HR. Transcranial surgery for pituitary adenomas. Neurosurgery 2005;57(01):168-175, discussion 168-175

31 Pallini R, Fernandez E, Lauretti L, et al. Olfactory groove meningioma: report of 99 cases surgically treated at the Catholic University School of Medicine, Rome. World Neurosurg 2015;83(02): 219-31.e1-219-31.e3 